\title{
Radiotherapy for unresectable locally advanced non-small cell lung cancer: a narrative review of the current landscape and future prospects in the era of immunotherapy
}

\author{
Tiantian Guo ${ }^{1,2 *}$, Liqing Zou ${ }^{1,2 *}$, Jianjiao $\mathrm{Ni}^{1,2}$, Xiao $\mathrm{Chu}^{1,2}$, Zhengfei $\mathrm{Zhu}^{1,2,3}$ \\ ${ }^{1}$ Department of Radiation Oncology, Fudan University Shanghai Cancer Center, Shanghai, China; ${ }^{2}$ Department of Oncology, Shanghai Medical \\ College, ${ }^{3}$ Institute of Thoracic Oncology, Fudan University, Shanghai, China \\ Contributions: (I) Conception and design: Z Zhu; (II) Administrative support: None; (III) Provision of study materials or patients: None; (IV) \\ Collection and assembly of data: All authors; (V) Data analysis and interpretation: All authors; (VI) Manuscript writing: All authors; (VII) Final \\ approval of manuscript: All authors. \\ "These two authors contributed equally to this work. \\ Correspondence to: Zhengfei Zhu, MD. Department of Radiation Oncology, Fudan University Shanghai Cancer Center, 270 Dong An Road, Shanghai, \\ 200032 China. Email: fuscczzf@163.com.
}

\begin{abstract}
Significant recent advances have occurred in the use of radiation therapy for locally advanced non-small cell lung cancer (LA-NSCLC). In fact, the past few decades have seen both therapeutic gains and setbacks in the evolution of radiotherapy for LA-NSCLC. The PACIFIC trial has heralded a new era of immunotherapy and has raised important questions for future study, such as the future directions of radiation therapy for LA-NSCLC in the era of immunotherapy. Modern radiotherapy techniques such as three-dimensional (3D) conformal radiotherapy and intensity-modulated radiotherapy (IMRT) provide opportunities for improved target conformity and reduced normal-tissue exposure. However, the low-dose radiation volume brought by IMRT and its effects on the immune system deserve particular attention when combing radiotherapy and immunotherapy. Particle radiotherapy offers dosimetric advantages and exhibits great immunoregulatory potential. With the ongoing improvement in particle radiotherapy techniques and knowledge, the combination of immunotherapy and particle radiotherapy has tremendous potential to improve treatment outcomes. Of particular importance are questions on the optimal radiation schedule in the settings of radio-immunotherapy. Strategies for the reduction of the irradiated field such as involvedfield irradiation (IFI) and omission of clinical target volume (CTV) hold promise for better preservation of immune function while not compromising locoregional and distant control. In addition, different dosefractionation regimens can have diverse effects on the immune system. Thus, prospective trials are urgently needed to establish the optimal dose fractionation regimen. Moreover, personalized radiotherapy which allows the tailoring of radiation dose to each individual's genetic background and immune state is of critical importance in maximizing the benefit of radiation to patients with LA-NSCLC.
\end{abstract}

Keywords: Immunotherapy; lung cancer; radiation therapy

Submitted Feb 25, 2020. Accepted for publication Jul 25, 2020.

doi: $10.21037 /$ tlcr-20-511

View this article at: http://dx.doi.org/10.21037/tlcr-20-511 


\section{Introduction}

Treatment of locally advanced non-small cell lung cancer (LA-NSCLC) is one of the greatest challenges facing oncologists. The role of chemotherapy and curativeintent radiotherapy is well recognized as the gold standard treatment for LA-NSCLC (1). Nonetheless, conventional treatment options for LA-NSCLC tend to reach a therapeutic plateau with suboptimal clinical outcomes $(2,3)$. Recently, the encouraging results of the PACIFIC trial, a multicenter randomized phase III trial of PD-L1 blockade durvalumab versus placebo in patients with non-progressive LA-NSCLC after concurrent chemoradiotherapy, have heralded a new era of immunotherapy in the treatment of LA-NSCLC (4). The combination of radiotherapy and immune checkpoint inhibitors (ICIs) significantly improved objective response rate, progression-free survival, and overall survival (OS), making it a new treatment paradigm in LA-NSCLC $(4,5)$.

The revolutionary advances in the treatment of LANSCLC are the result of the advent of immunotherapy, which offers opportunities to augment antitumor immunity (6). Prominent recent progress in immunotherapies for NSCLC includes the development of ICIs (e.g., anti-CTLA-4 antibodies and anti-PD-1/PD-L1 antibodies), cytokines and cytokine blockers (e.g., GM-CSF, IL-2, and TGF- $\beta$ blockade), oncolytic viruses (e.g., ADV/HSV-tk), and other targeted immunotherapies (e.g., OX-40 antibodies, Tolllike receptors (TLR) agonists, and IOD1 inhibitors) (6-11). To date, PD-1 inhibitors pembrolizumab and nivolumab, PD-L1 inhibitor atezolizumab, and CTLA-4 blockade ipilimumab combined with nivolumab have demonstrated impressive efficacy in prospective trials and are approved by the FDA for the treatment of metastatic NSCLC (12-15).

Meanwhile, we are also in the midst of one of the most exciting times of radiation oncology, in which technological advances are now enabling more accurate radiation delivery with limited exposure of surrounding normal tissues. The integration of radiotherapy with immunotherapy is expected to revolutionize cancer treatment, given that numerous murine studies have shown the synergistic antitumor effect of this combination strategy (16-18). Mechanistically, radiation can, via various mechanisms, stimulate antitumor immune response (6). For example, radiation-induced immunogenic cell death triggers the release of tumor antigens and damage-associated molecular patterns (DAMPs) and the production of type I interferons (IFNs) $(19,20)$. Moreover, radiation initiates the production of pro-inflammatory cytokines, such as tumor necrosis factor (TNF) and interleukin $1 \beta$ (IL-1 $\beta$ ), which triggers the infiltration of immune cells (e.g., dendritic cells and cytotoxic $\mathrm{T}$ cells) and results in an inflammatory tumor microenvironment (21-23). Radiation also enhances systemic immune activation which manifests itself as the abscopal effect, where tumor regression occurs in nonirradiated sites (24). This phenomenon is presumably attributable to the capacity of radiotherapy to convert the tumor into an in situ vaccine (25). Additionally, radiation induces the upregulation of PD-L1 on tumor cells (26), and thus integrating radiotherapy with antiPD1/PD-L1 antibodies can overcome adaptive immune resistance. Notably, the use of immunotherapy in nonmetastatic settings has recently garnered attention as growing preclinical data support the potential utility for ICIs to reduce metastatic relapse from localized disease $(27,28)$. Furthermore, based on the strong evidence from the PACIFIC trial, PD-L1 inhibitor durvalumab has been approved by the FDA for patients with non-progressive LA-NSCLC after concurrent chemoradiotherapy. The success of the PACIFIC trial has increased enthusiasm for the integration of radiotherapy with immunotherapy for the treatment of LA-NSCLC.

However, radiotherapy is a double-edged sword to immunotherapy, involving not only the enhanced antitumor activity but also increased normal tissue toxicities and risks of lymphopenia. Radiation-induced normal tissue injury begins with a cascade of molecular events, including reactive oxygen species production and DNA damage (29). The subsequent release of DAMPs induces the release of proinflammatory cytokines (e.g., IL-1 and TNF- $\alpha$ ) through the activation of the nuclear factor kappa-B (NF- $\mathrm{\kappa B})$ signaling pathway (30), leading to activation of resident immune cells and recruitment of pro-inflammatory cells (31). Given that immunotherapy helps boost the immune system, the combination of radiation with immunotherapy may increase the risk of normal tissue toxicities. In the PACIFIC trial (4), although the combination of radiotherapy and immunotherapy did not increase the incidence of serious side effects ( $\geq$ grade 3 ) such as pneumonitis, it increased the incidence of toxicities at all grades. Among the toxicities caused by the thoracic radiation plus ICI regimen, the most common studied overlapping toxicities are cardiotoxicity and pulmonary toxicity (32-34). In fact, both radiotherapy and immunotherapy carry the potential risk of cardiotoxicity and pulmonary toxicity (35-38). The synergistic interaction between immunotherapy and thoracic irradiation in 
increasing the risk of pulmonary and cardiotoxicities has been proven in series preclinical models $(32,33)$. In addition, radiation exposure is also known to have suppressive effects on the immune system (39). Lymphocytes, as part of systematic immune cells, are extremely sensitive to radiation exposure (40), and a single radiation dose of 1-3 Gy has been demonstrated to induce apoptosis in lymphocytes (41). Research has shown that radiation-induced lymphopenia (RIL) occurs in $40 \%$ to $70 \%$ of patients treated with radiotherapy, potentially attributed to the direct irradiation of lymph nodes and to circulating lymphocytes (CLs) traversing through the radiation field (42). Lymphocytes are heavily involved in the antitumor activity of immune system. The nadir of absolute lymphocyte counts (ALC) during radiotherapy has been shown to be associated with worse survival in NSCLC patients in multiple studies (43-45), and the depletion of lymphocytes is potentially linked with a lower likelihood of response to immunotherapy for NSCLC (46). Therefore, special attention is needed to better preserve lymphocyte function when combining immunotherapy with radiotherapy.

Since the pre-immunotherapy era, radiation therapy has evolved significantly. In fact, the past few decades have witnessed both therapeutic gains and setbacks in the development of radiotherapy for LA-NSCLC. The PACIFIC trial has opened new horizons for the management of LA-NSCLC, but has also raised important questions, including the future directions of radiation therapy for LA-NSCLC in the era of immunotherapy. Current radiotherapy for LA-NSCLC is not yet fully optimized for this combination strategy with many unanswered questions, including the appropriate choice of radiation techniques, appropriate radiation target volumes, and appropriate dose-fractionation regimen to combine with immunotherapy. Here, we review the current data with regard to the radiotherapy techniques (including photon-based radiotherapy and particle beam therapy) and strategies (including reduction of radiation target volumes and dose-fractionation regimen) for the treatment of LANSCLC with a particular focus on the future directions and challenges for radiotherapy in the era of immunotherapy. A literature search in MEDLINE and EMBASE was conducted (date of the last search 15 January 2020) to identify English-language publications on radiotherapy for LA-NSCLC and its combination with immunotherapy, supplemented by manual searches of the reference lists of identified articles and relevant reviews.

We present the following article in accordance with the Narrative Review checklist (available at http://dx.doi. org/10.21037/tlcr-20-511).

\section{Radiation techniques}

\section{Photon-based radiotherapy}

The paradigm shift from two-dimensional radiotherapy (2DRT) to advanced three-dimensional (3D)-based radiation techniques, including 3 -dimensional conformal radiotherapy (3DCRT) and intensity-modulated radiotherapy (IMRT), allows for more accurate radiation delivery and limited exposure of adjacent critical structures. The theoretical advantages of 3DCRT mainly lie in its superior conformity in using computed tomography (CT) for treatment planning, considering the larger target volumes caused by the deficiency in precise visualization of target lesions on a $2 \mathrm{D}$ radiograph. IMRT can currently be delivered in static mode (fixed-field IMRT), volumetricmodulated arc therapy (VMAT), helical tomotherapy (HT), and other modalities. All forms of IMRT enable the intensity modulation of each beam and further improve target conformity with a substantial decrease in the doses to normal tissues (47-49). Consequently, in contrast to 2DRT, 3D-based radiation techniques can achieve improvement of local control with lower rates of treatment-related toxicity.

The evolution of radiation techniques from 2DRT to 3DCRT and to IMRT in turn, have revolutionized the treatment of LA-NSCLC. A National Cancer Data Base $(\mathrm{NCDB})$ analysis reported that $3 \mathrm{D}$-based radiation techniques, including 3DCRT and IMRT, were associated with a significantly improved OS compared with conventional 2DRT (3-year OS 22\% vs. 19\%; 5 -year OS $14 \%$ vs. $11 \%, \mathrm{P}<0.0001)(50)$. In terms of treatment-related toxicity, Yom and colleagues demonstrated a substantial reduction in the rates of grade $\geq 3$ radiation pneumonitis at 1 year in patients treated with IMRT compared with 3DCRT ( $8 \%$ vs. $32 \%, \mathrm{P}=0.002$ ), despite the larger gross tumor volume (GTV) in the IMRT cohort (51). Liao and colleagues evaluated disease outcomes and rates of toxicity in patients treated with IMRT or 3DCRT combined with concurrent chemotherapy. OS for the IMRT group was superior to that of the 3DCRT group. Moreover, IMRT was associated with reduced rates of grade $\geq 3$ radiation pneumonitis (52). Notably, given that the median radiation doses of 63 Gy were the same for both groups in this study (52), the survival benefit of IMRT over 3DCRT may be mainly attributed to reduction in doses to normal 
structures and the consequent striking improvements in toxicity profiles. Reducing toxicity is a constantly recurring theme in the field of radiotherapy. Especially in the era of immunotherapy, given the synergistic toxicity of combined radiotherapy and immunotherapy, advanced radiation techniques for safer and more accurate radiation delivery will offer opportunities to reduce rates of toxicity and elevate the success of cancer treatment to a new level when combined with immunotherapy.

However, it is worth noting that improved sparing of organs at risk (OAR) of IMRT might come at the expense of more area being exposed to low-dose radiation. In fact, planning studies have shown that lung volume $>5$ Gy (V5) increased in the IMRT plans compared with 3DCRT plans $(49,53)$. Another comparative study on the dosimetric features between fixed-field IMRT and HT reported significantly reduced lung V20-30 in the HT plan, together with larger volumes of low dose radiation (lung V5-10) (54). In the pre-immunotherapy era, multiple studies have focused on the potentially increased risk of pulmonary toxicity resulting from larger volumes of normal lung exposed to low-dose radiation (55). Two retrospective studies from the MD Anderson Cancer Center demonstrated that lung V5 was significantly associated with pneumonitis risks in patients receiving concurrent chemoradiotherapy (56,57). Apart from pulmonary toxicity, the risk of lymphopenia caused by the low-dose irradiation effect of IMRT will be of paramount concern in the era of immunotherapy. Tang and colleagues reported that lower dose radiation volume, especially lung V5-10, exhibited a greater association with lymphocyte nadirs than high dose ranges (43). Given the association of lower lymphocytes with poorer clinical outcomes $(43,44,58)$ and the lower likelihood of response to immunotherapy (46), special attention should be given to the low-dose radiation volume offered by IMRT in the era of immunotherapy.

\section{Particle beam therapy}

Particle radiotherapy, which mainly includes proton and carbon-ion radiotherapy, has experienced a surge in attention as another promising treatment modality for LANSCLC. Compared with photon radiotherapy, the primary strength of particle therapy lies in its dose distribution capabilities. The particles release little energy during travel and deposit most of their energy near the end of their path, which is known as the Bragg peak phenomenon. The unique dose distribution characteristics allow for highly conformal and high-dose delivery to the tumor and the sparing of surrounding normal tissues. These qualities therefore make particle radiotherapy an attractive treatment option for LANSCLC in which the target lesion typically lies in close proximity to vital organs such as the heart, spinal cord, and esophagus. Multiple dosimetric studies have demonstrated the superiority of proton therapy over photon therapy in reducing volumes of normal lung tissues receiving low dose ranges and constraining doses for critical structures such as the heart, esophagus, and spinal cord $(59,60)$.

The dosimetric advantages of proton therapy are of particular interest in the burgeoning field of combining cancer immunotherapy and radiotherapy. Currently, uncertainty remains over the potential of proton therapy for better clinical outcomes compared with IMRT in patients with LA-NSCLC. A large retrospective study of the National Cancer Database showed that proton therapy conferred a significant OS benefit in stage II and III NSCLC patients compared with photon therapy. However, the OS difference failed to reach statistical significance in propensity score-matched cohorts (61). In addition, a recent prospective randomized phase II clinical trial comparing passively scattered proton therapy (PSPT) and IMRT failed to demonstrate the advantages of proton therapy over IMRT in terms of reducing toxicity, showing no significant differences in the rates of grade $\geq 3$ radiation pneumonitis or local failure (62). Larger volumes exposed to higher doses (20$80 \mathrm{~Gy}$ ) and lack of sufficient experience in proton radiation planning may account for the negative clinical outcomes (62). Of note, proton therapy is currently delivered either using PSPT, or pencil-beam scanning (PBS). Compared with PSPT, PBS offers the advantage of better dose distribution and greater sparing of normal structures compared with PSPT (59). However, the uncertainties associated with the range of the proton beam and respiratory motion, along with the tissue density heterogeneity of chest organs, also render implementing PBS proton therapy for LA-NSCLC more challenging and demanding (63).

With the ongoing improvement of proton therapy technique and knowledge, we believe that proton therapy still has great potential to enhance treatment outcome when combined with immunotherapy. First, proton radiotherapy displays the dosimetric advantage of reduced radiation doses for critical organs, especially in heart exposure, which may confer a long-term survival benefit (62). Second, some research has shown the superiority of proton therapy over photon therapy in reducing the risks of grade 4 lymphopenia $(64,65)$. Reduced volume of low-dose radiation could limit the 
radiation exposure of peripheral blood lymphocytes, and thus may serve to increase the efficacy of immunotherapy through lymphocyte sparing. Thus, future randomized studies are needed to show whether the combination of immunotherapy with proton therapy, especially PBS proton therapy, will lead to improved outcomes for patients with LA-NSCLC.

In addition to the above-mentioned dosimetric advantage, particle radiotherapy also exhibits great immunoregulatory potential. Gameiro and colleagues reported that protons significantly down-regulated PD-L1 and induced higher levels of calreticulin expression on the tumor cell surface than photons in different tumor cell lines (66). This result gives support to the promise of proton radiotherapy leading to enhanced T-cell mediated antitumor activity in irradiated tumors, and thus suggests an emerging role for proton therapy in facilitating antitumor response when combined with T-cell mediated immunotherapy. Dendritic cells (DC) serve as specialized antigen-presenting cells and play a pivotal role in initiating antitumor immune response after uptake of tumor antigens (67). Accumulating evidence from preclinical studies indicates that carbon-ion irradiation in combination with DC injection has anti-metastatic effects. Ohkubo and colleagues reported that, compared with carbon-ion irradiation alone, combined carbon-ion irradiation and intratumoral DC injection correlated with a significant decrease in the number of lung metastases in a mouse model (68). In another preclinical study, a significantly enhanced anti-metastatic effect was observed with the combination of low dose carbon-ion irradiation and DC injection, while the combination of photon irradiation required a higher dose to suppress tumor metastasis (69). Moreover, in combination with carbon-ion irradiation, intravenous DC injection was more effective in suppressing lung metastases than intratumoral DC administration (69). In clinical practice, intravenous DC injection is also more suitable with respect to the advantage of carbon-ions in treating deep-seated tumors. The enhanced immunogenicity of tumor cells with up-regulation of calreticulin on cell surfaces and the activation of immature DCs during carbonion irradiation may contribute to a synergic interaction between carbon-ion radiotherapy and immunotherapy $(69,70)$.

\section{Reduction of radiation target volumes}

\section{Elective node irradiation (ENI)}

ENI in NSCLC refers to the radiation of lymph nodes that have not metastasized according to clinical judgment, and can include the bilateral hilar, mediastinum, and even supraclavicular areas. This treatment was originally intended as a means to kill subclinical lesions that may exist in these areas. However, the idea of ENI was later questioned because it was found to increase the volume of the radiotherapy target, thus leading to toxicity and making it hard to improve the therapeutic dose (47). Indeed, in the pre-immunotherapy era, accumulated evidence has suggested that involved-field irradiation (IFI) instead of ENI is a better treatment strategy for LA-NSCLC. A prospective randomized study (71) compared IFI versus ENI for patients with inoperable stage III NSCLC treated with concurrent chemoradiotherapy. 3DCRT was delivered with $1.8-2$ Gy/Fx to $68-74$ Gy for the IFI arm and with 60-64 Gy for the ENI arm. The results showed that the IFI arm achieved a better OS rate $(90 \%$ vs. $79 \%, \mathrm{P}=0.032)$ and a better 5 -year local control rate (LCR) $(51 \%$ vs. $36 \%$, $\mathrm{P}=0.032)$ than the ENI arm. Despite the higher dose, the radiation pneumonitis rate in patients with IFI was actually lower than that in patients with ENI (17\% vs. 29\%, $\mathrm{P}=0.044)$. However, given the higher prescribed dose in the IFI arm, whether the better outcome was due to the higher radiation dose or due to IFI, is still disputed. Notably, in another prospective cohort comparing IFI to ENI, a maximum radiation dose, given the condition that OAR could be tolerated in both arms, was given by Chen and colleagues who found a tendency of improved locoregional PFS rate with IFI ( $34.1 \%$ vs. $30 \%, \mathrm{P}=0.673)$, along with a significant increased OS rate (72). Li and colleagues performed a meta-analysis comprising 3 RCTs and 3 cohort studies to compare the incidence of elective nodal failure (ENF) in ENI versus IFI. The results showed no significant difference in the incidence of ENF between IFI and ENI either among RCTs $(\mathrm{P}=0.46)$ or cohort studies $(\mathrm{P}=0.97)$, or when RCTs and cohort studies were combined $(\mathrm{P}=0.64)(73)$. Additionally, numerous retrospective studies of patients with stage III NSCLC treated with definitive radiotherapy have reported that IFI was associated with an acceptably low ENF rate and a significantly lower risk of higher-grade esophagitis (74-76).

In the era of immunotherapy, in addition to OS, localregional control, and toxicity profile, the interaction between radiation and the immune system needs to be further considered when we choose between IFI and ENI. The tumor-immune cycle is divided into the following 7 steps: (I) tumor antigen release, (II) tumor antigen presentation, (III) activation of effector T cells, (IV) 
migration of $\mathrm{T}$ cells to tumor tissues, $(\mathrm{V})$ tumor tissue $\mathrm{T}$ cell infiltration, (VI) recognition of tumor cells by $\mathrm{T}$ cells, and (VII) removal of tumor cells (77). Obviously, abnormalities in any one of these steps can lead to an antitumor-immune cycle failure and an immune escape, which emphasizes the importance of the integrity of the lymphatic system in the efficacy of radiotherapy plus immunotherapy. Previous studies have demonstrated that direct irradiation of lymph nodes can partially explain lymphopenia, since lymph nodes act as reservoirs of lymphocytes and also as depots for clonal expansion of lymphocytes to specific antigens (78). Additionally, Tang and colleagues reported that larger GTVs exhibited a significant association with lymphocyte nadirs (43). In the case of locally advanced NSCLC for which thoracic radiation is needed, it is especially noteworthy that CLs receive a significant dose of radiation during the passes through the heart, and the entire cardiac output also transits through the pulmonary circulation, thus making it more easily affected by radiation (78). Moreover, there is emerging evidence indicating that the depletion of lymphocytes is linked with poorer outcomes in NSCLC patients $(43,44,58)$ and, notably in the era of immunotherapy, a lower likelihood of response to immunotherapy (46). In preclinical studies, stereotactic radiotherapy with ENI, in combination with immune checkpoint blockade (ICB), restrained immune infiltration, attenuated chemokine expression, and negatively correlated with survival (79).

Overall, IFI can achieve better normal tissue sparing and less toxicity while not compromising locoregional and distant control. More importantly, based on preclinical and clinical evidence, IFI may better synergize with immunotherapy than ENI, thus making it part of powerful radiotherapy strategy in the era of immunotherapy.

\section{Omission of clinical target volume (CTV)}

In addition to IFI, omission of CTV is another strategy for reducing target volume. Traditionally, the planning target volume (PTV) was generated by expanding step by step from GTV, CTV, and internal target volume (ITV), and then the radical radiation dose was prescribed to the PTV in the definitive radiotherapy for LA-NSCLC. However, the CTV is defined as the tissue volume that contains the GTV and subclinical microscopic malignant lesions, and several studies have proven that the doses needed to eradicate subclinical diseases are lower than those used to control gross tumors in patients with common epithelial tumors
(80-82). From this point of view, the dosage prescribed to the subclinical lesions in the traditional target-contouring might be higher than needed. Previous experience has led to the conclusion that radiation doses of 45-50 Gy could result in high control rates for subclinical disease in patients with epithelial tumors $(80,81)$. With this in mind, we performed a dosimetric study on LA-NSCLC (83) and found that radiotherapy with the IMRT technique could deliver sufficient dose coverage to subclinical regions while reducing the dose to normal tissues when CTV was omitted. CTV omission has also been proven to be acceptable in clinical settings. A retrospective study of 105 stage III NSCLC patients treated with $(n=50)$ or without CTV $(n=55)$ revealed no statistical significance in terms of local recurrence, distant metastasis, progression-free survival (PFS), OS, and grade 3-4 radiation esophagitis, or hematological toxicity between 2 two arms. Notably, the grade 3-4 radiation pneumonia rate was significantly lower in the arm without CTV $(\mathrm{P}=0.044)(84)$. Kilburn and colleagues evaluated recurrence sites of 110 stage IIIII NSCLC patients treated without CTV and found only 2 CTVretro (PTVs expanded $1 \mathrm{~cm}$ ) failures, thus indicating that CTV omission appears to be a feasible strategy (85). It is worth noting that larger target volume not only increases the risk of radiation toxicities but can also cause more damage to the lymphatic system. In this regard, omission of CTV has the potential to better preserve lymphocyte function and holds great promise for better synergetic effects when combined with immunotherapy.

\section{Radiation dose and fractionation}

\section{Dose escalation and altered fractionation}

In the pre-immunotherapy era, the standard radiation dose for unresectable LA-NSCLC was 60-63 Gy in 1.8-2 Gy daily fractions, which was established by the RTOG 7301 trial in 1980 (86). The strategy of dose escalation has shown surprisingly disappointing results in RTOG 0617 as dose escalation from 60 to 74 Gy resulted in worse OS compared to $60 \mathrm{~Gy}$ (87). Besides dose escalation, considerable interest has been focused on exploring the potential benefits of unconventional fractionation radiotherapy for the treatment of LA-NSCLC. An individual patient data meta-analysis has shown that hyper-fractionated and accelerated radiotherapy yielded a modest survival benefit in LA-NSCLC patients compared with conventional schedules (88). However, it is worth noting that among all the included trials in this meta- 
analysis, the benefit in OS reached statistical significance only in the continuous hyper-fractionated accelerated radiotherapy (CHART) trial, in which patients received radiotherapy without chemotherapy (89). Meanwhile, numerous other phase III trials reported that the hyperfractionated/accelerated radiotherapy regimen did not achieve significant survival advantage over conventional radiotherapy when combined with induction or concurrent chemotherapy (90-93).

In the era of immunotherapy, the optimal dosefractionation regimen remains to be clarified. The current dose-fractionation regimen is based on the linearquadratic (LQ) model, which calculates the optimal dosefraction to eliminate a certain type of tumor while sparing its surrounding normal tissues. However, the LQ model only focuses on the radiation killing of tumor cells, while neglecting the role of the immune system in antitumor activity. The immune system is of critical importance to tumor control after radiotherapy, while radiation exposure can have destructive effects on lymphocytes $(40,94,95)$. Increased total dose, lung V5, twice-daily fractionation, and extended radiotherapy duration were found as risk factors for lymphopenia. On the other hand, enhanced antigen presentation is another known effect of radiotherapy (96-99). Varying doses of radiation (1-100 Gy) in a single fraction or in a short-course fraction regimen can induce diverse immunogenic effects. A recent preclinical study demonstrated that hypo-fractionated radiation was more immunogenic compared to the conventional fractionated regimen (100). Vanpouille-Box and colleagues found that radiation doses above a certain threshold (12-18 Gy) attenuated tumor immunogenicity by inducing DNA exonuclease Trex1 and degrading cytosolic DNA (101), thus suggesting that fractionated doses should not surpass the threshold for Trex1 induction.

In addition, one of the hurdles to the success of immunotherapy is immunosuppressive tumor microenvironment (TME). Both conventional fractionation radiotherapy and stereotactic body radiotherapy (SBRT) can "de-bulk" the tumor $(23,102)$, thus leading to direct destruction of the TME. Radiation also induces phenotypic changes in immunosuppressive cell populations in the TME including myeloid-derived suppressor cells (MDSCs), M2 tumor-associated macrophages (TAMs), and regulatory $\mathrm{T}$ (Treg) cells. For example, radiation has been reported to induce the infiltration of M2-like TAMs (103-105), which display pro-survival and pro-angiogenic activities, whereas other studies have shown that low-dose radiation
(2 Gy 1-2 fx daily) promotes the polarization of TAMs from $\mathrm{M} 2$ to NOS+ M1 and improves anti-tumor immune response $(106,107)$. Filatenkov and colleagues reported that immunosuppressive TME was transformed by a single 30 Gy dose of radiation that decreased the infiltration of MDSCs (108). In contrast, the addition of conventional daily fractionated (10×3 Gy) to the single dose of 30 Gy resulted in significantly increased infiltration of MDSCs (108). Moreover, Lan and colleagues found that compared with conventional daily low-dose fractionated radiotherapy, hypo-fractionated radiotherapy inhibited hypoxia within primary tumors, decreased vascular endothelial growth factor (VEGF) expression, and reduced the recruitment of MDSCs into tumors (109).

Taken together, these findings suggest that different dose-fractionation regimens can have diverse effects on the immune system. Thus, in the era of immunotherapy, the dose-fractionation schedules should be re-evaluated to determine the optimal radiation regimen. Notably, the combination of ICB and non-conventional dose fractionation radiotherapy is currently being explored by several ongoing clinical investigations (NCT04081688; NCT03801902; NCT03589547; NCT03237377).

\section{Individualized radiation dose}

The development of precision medicine has revolutionized systemic therapy for lung malignancies. To date, although accumulating evidence has demonstrated considerable heterogeneity of radiosensitivity and radiotoxicity between patients with the same tumor histology and disease stage but different molecular background (110-113), radiation dose protocols are still uniform for all LA-NSCLC patients (one-size-fit-all).

Tailoring of the radiation dose to individualized patienttumor radiosensitivity holds great promise as an effective radiotherapy strategy. Radiosensitivity of tumor cells can be strongly impacted by molecular variations on the genomic, transcriptional, and translational levels. Genetic mutations or single nucleotide polymorphisms (SNPs) of DNA repair response-associated genes (such as p53, ATM, BRCA1, BRCA2, ERCC1, XRCC3, and Rad51) have been repeatedly found to be associated with radiosensitivity in lung cancer (114-118). In addition, mutations or SNPs of crucial oncogenes (such as EGFR and ALK) (119-122) or critical radiation-modulating genes (such as TGF- $\beta$ ) (123-125) have recently been shown to influence tumor radiosensitivity. Moreover, comprehensive analyses of 
radiosensitivity-associated genes and proteins in lung cancer $(126)$ and other solid tumors $(127,128)$ have been carried out to identify potential biological predictors of radiosensitivity. Accompanying the gradually deepening understanding of the mechanisms and biomarkers of radiosensitivity, gene-expression classifiers that incorporate a handful of vital genes to predict radiosensitivity in specific tumor types or across various human cancers have become available $(129,130)$.

Genomic-adjusted radiation dose (GARD), one of the most prominent radio-sensitivity prediction algorithms, was derived from the gene-expression-based radiationsensitivity index and the linear quadratic model. A total of 10 genes were identified and validated by training a linear regression model to predict the experimental survival fraction at 2 Gy for 48 cancer cell lines from 9 different disease sites $(129,131,132)$. Afterward, the radiosensitivity index (RSI) was calculated using a mathematic algorithm incorporating all of 10 genes with distinct weights. Finally, the GARD score was calculated using the linear quadratic model, the individual RSI, and the standard radiation dose/ fractionation. GARD scores of patients from 5 different cohorts consisting of primary tumors from 20 disease sites were measured and demonstrated to be independently associated with clinical outcome in breast cancer, lung cancer, glioblastoma, and pancreatic cancer (133). Based on these data, an individualized radiation dose could be suggested both for the primary tumors (134) and lymph nodes (135). However, the GARD score has not been widely used in clinical practice due to the lack of sufficient validation in randomized clinical trials.

In addition, radiation-induced toxicity, which is mainly determined by the biologic and genetic background of surrounding normal tissues, should also be taken into consideration when personalizing radiation dose. A list of germline SNPs has been found to be associated with acute and long-term radiotoxicity $(112,136,137)$. Chemotherapy (especially irinotecan) dose modification according to highly recognized SNPs has been validated in several clinical trials among metastatic patients receiving chemotherapy alone or locally advanced patients receiving chemoradiotherapy (138-140). Similarly, personalized radiotherapy strategies such as the tailoring of the radiation dose to each individual's genetic background aimed to reduce radiationinduced toxicity are particularly attractive.

In the era of immunotherapy, particular attention should be paid to the tumor immune microenvironment and systematic immune state when personalizing the radiation dose, since the potency and durability of the anti-tumor immune response induced by radiotherapy are significantly influenced by these critical factors $(101,141)$. In preclinical models, the radiation dose greater than 12 Gy could attenuate the anti-tumor immune response by degrading DNA that accumulated in the cytosol upon radiation. On the other hand, an enhanced anti-tumor immune response was observed when radiation dose was elevated in the range of 0-8 Gy (101). Therefore, hypo-fractionated radiation at a dose of 5-10 Gy per fraction was speculated to be better than conventionally fractionated schemes of 1.8-2.2 Gy fractions (141). In fact, prospective clinical trials evaluating the combination of anti-PD-1 blockade with SBRT at a dose in that range for the treatment of metastatic lung cancer have yielded promising results $(142,143)$. However, low-dose per-fraction radiation was found to recruit more cytotoxic T cells into the TME than high doses in another preclinical study (106). One recent retrospective study showed that higher doses (estimated dose of radiation to immune cells larger than $6.1 \mathrm{~Gy}$ ) of radiation to the immune system were associated with tumor progression and death after the definitive treatment of stage III NSCLC (143). Additionally, one systematic analysis of patients treated with radiation and ipilimumab demonstrated that low fractional doses of radiation were associated with a more favorable systemic response (144). Taken together, the optimal radiation dose in the settings of radio-immunotherapy remains controversial (142), and it is likely to be dependent on biological features of tumor cells and the immune state of each individual patient. A number of questions need to be answered before we can prescribe an individualized radiation dose in the hope of provoking the most potent and durable anti-tumor immune response.

\section{Conclusions and future directions}

The era of immunotherapy is destined to be a time of great challenge but also wonderful opportunity for the use of radiation therapy for LA-NSCLC. Immune-sparing strategies and the minimization of radiation exposure to adjacent critical structures will be key to a successful combination of thoracic radiotherapy and immunotherapy (Figure 1). Advanced radiotherapy techniques such as 3DCRT and IMRT offer opportunities for improved target conformity and reduced normal-tissue exposure. However, the low-dose radiation volume brought by IMRT and its effects on the immune system deserve particular attention. The dosimetric advantages and immunoregulatory potential 


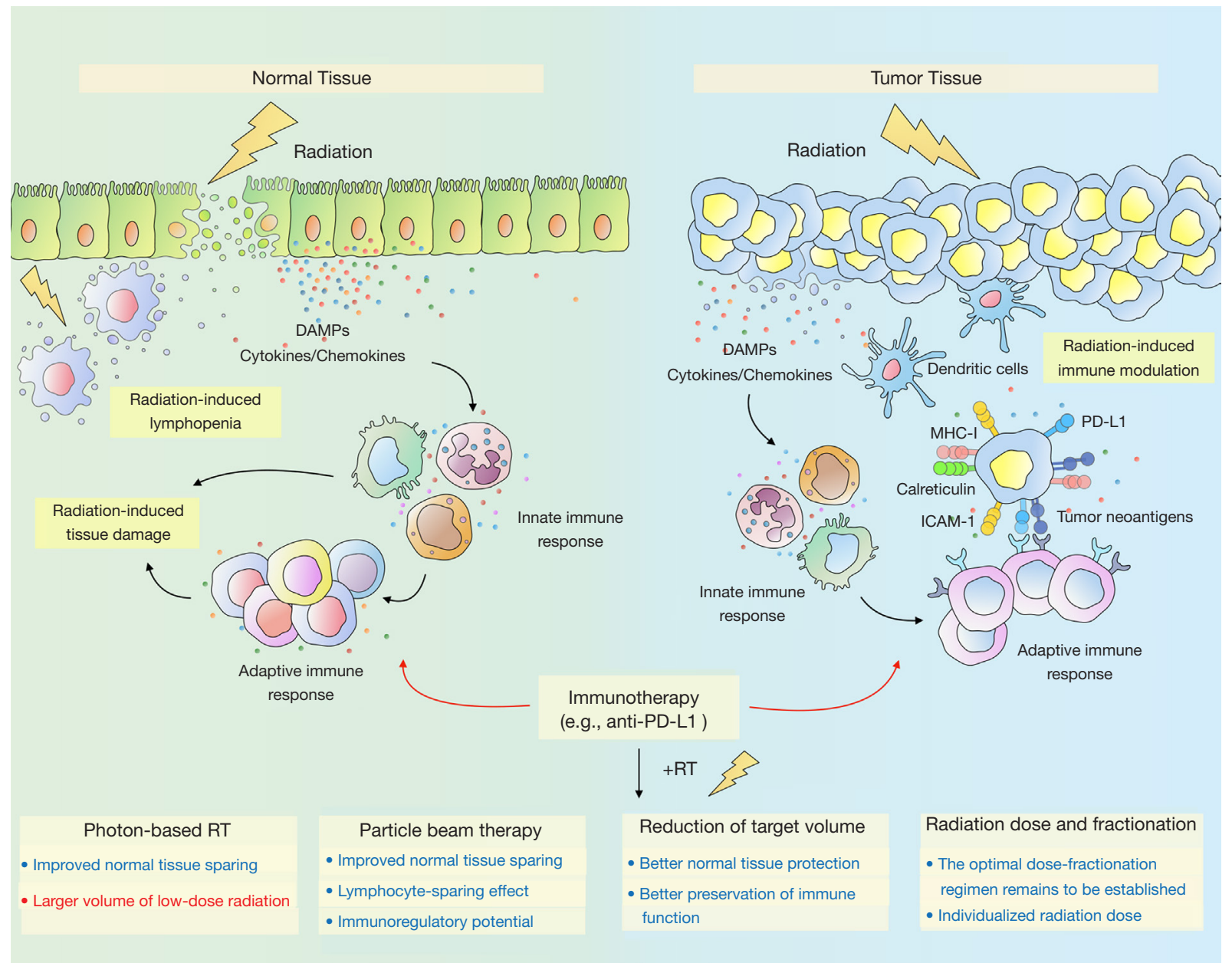

Figure 1 Schematic representation showing radiation-induced immune modulation in the tumor microenvironment and radiationinduced normal tissue toxicity. Radiation can augment antitumor immune response through diverse mechanisms. For example, radiation stimulates the release of tumor antigens and DAMPs, initiates the production of pro-inflammatory cytokines, and triggers the upregulation of immunomodulatory surface molecules (e.g., ICAM-1 and MHC-I), leading to the infiltration of immune cells such as dendritic cells and cytotoxic T cells. Additionally, radiation induces the upregulation of PD-L1 on tumor cells, and thus integrating radiotherapy with antiPD1/PD-L1 antibodies can overcome this resistance mechanism. Different dose-fractionation regimens can have diverse effects on the immune system. Further investigation is needed to establish the optimal radiation regimen. On the other hand, radiation-induced damage to tissue-resident cells triggers the release of DAMPs and pro-inflammatory mediators, allowing activation of resident immune cells and recruitment of inflammatory cells, which, in turn, amplifies the ongoing inflammatory response. Moreover, radiation can have destructive effects on lymphocytes and result in radiation-induced lymphopenia. Thus, immune-sparing strategies and the minimization of radiation exposure to normal tissues will be key to a successful combination of radiotherapy and immunotherapy. DAMPs, damage-associated molecular patterns; ICAM-1, intercellular cell adhesion molecule-1; MHC-I, major histocompatibility complex class I; PD-1, programmed cell death protein-1; PD-L1, programmed death ligand 1; RT, radiotherapy.

of particle radiotherapy make it an attractive option in the era of immunotherapy, which merits further investigation. Strategies for the reduction of radiation target volumes such as IFI and CTV omission can achieve better normal tissuesparing while not compromising locoregional and distant control. More importantly, both IFI and CTV omission could better preserve immune function. Thus, there is immense potential for IFI and omission of CTV to achieve better synergetic effects in the era of immunotherapy. The optimal dose fractionation regimen in the settings of 
radio-immunotherapy remains to be established in future prospective trials. Personalized radiotherapy might be of particular interest in allowing the administration of individualized radiation dose according to each patient's genetic background and immune state.

\section{Acknowledgments}

Funding: This project was supported by the National Science Foundation of China (No. 81572963 to Zhengfei Zhu).

\section{Footnote}

Reporting Checklist: The authors have completed the Narrative Review checklist. Available at http://dx.doi. org/10.21037/tlcr-20-511

Conflicts of Interest: All authors have completed the ICMJE uniform disclosure form (available at http://dx.doi. org/10.21037/tlcr-20-511). Dr. ZZ reports receiving consulting fees and honoraria from AstraZeneca. The other authors have no conflicts of interest to declare.

Ethical Statement: The authors are accountable for all aspects of the work in ensuring that questions related to the accuracy or integrity of any part of the work are appropriately investigated and resolved.

Open Access Statement: This is an Open Access article distributed in accordance with the Creative Commons Attribution-NonCommercial-NoDerivs 4.0 International License (CC BY-NC-ND 4.0), which permits the noncommercial replication and distribution of the article with the strict proviso that no changes or edits are made and the original work is properly cited (including links to both the formal publication through the relevant DOI and the license). See: https://creativecommons.org/licenses/by-nc-nd/4.0/.

\section{References}

1. Eberhardt WE, De Ruysscher D, Weder W, et al. 2nd ESMO Consensus Conference in Lung Cancer: locally advanced stage III non-small-cell lung cancer. Ann Oncol 2015;26:1573-88.

2. Machtay M, Paulus R, Moughan J, et al. Defining local-regional control and its importance in locally advanced non-small cell lung carcinoma. J Thorac Oncol
2012;7:716-22.

3. Auperin A, Le Pechoux C, Rolland E, et al. Meta-analysis of concomitant versus sequential radiochemotherapy in locally advanced non-small-cell lung cancer. J Clin Oncol 2010;28:2181-90.

4. Antonia SJ, Villegas A, Daniel D, et al. Durvalumab after Chemoradiotherapy in Stage III Non-Small-Cell Lung Cancer. N Engl J Med 2017;377:1919-29.

5. Antonia SJ, Villegas A, Daniel D, et al. Overall Survival with Durvalumab after Chemoradiotherapy in Stage III NSCLC. N Engl J Med 2018;379:2342-50.

6. Chae YK, Arya A, Iams W, et al. Immune checkpoint pathways in non-small cell lung cancer. Ann Transl Med 2018;6:88.

7. Tay R, Prelaj A, Califano R. Immune checkpoint blockade for advanced non-small cell lung cancer: challenging clinical scenarios. J Thorac Dis 2018;10:S1494-502.

8. Aspeslagh S, Postel-Vinay S, Rusakiewicz S, et al. Rationale for anti-OX40 cancer immunotherapy. Eur J Cancer 2016;52:50-66.

9. Kaczanowska S, Joseph AM, Davila E. TLR agonists: our best frenemy in cancer immunotherapy. J Leukoc Biol 2013;93:847-63.

10. Yue EW, Sparks R, Polam P, et al. INCB24360 (Epacadostat), a Highly Potent and Selective Indoleamine2,3-dioxygenase 1 (IDO1) Inhibitor for Immuno-oncology. ACS Med Chem Lett 2017;8:486-91.

11. Kang J, Demaria S, Formenti S. Current clinical trials testing the combination of immunotherapy with radiotherapy. J Immunother Cancer 2016;4:51.

12. Rittmeyer A, Barlesi F, Waterkamp D, et al. Atezolizumab versus docetaxel in patients with previously treated non-small-cell lung cancer (OAK): a phase 3, openlabel, multicentre randomised controlled trial. Lancet 2017;389:255-65.

13. Hellmann MD, Paz-Ares L, Bernabe Caro R, et al. Nivolumab plus Ipilimumab in Advanced Non-Small-Cell Lung Cancer. N Engl J Med 2019;381:2020-31.

14. Reck M, Rodríguez-Abreu D, Robinson AG, et al. Pembrolizumab versus Chemotherapy for PD-L1Positive Non-Small-Cell Lung Cancer. N Engl J Med 2016;375:1823-33.

15. Jørgensen JT, Nielsen KB. Companion and complementary diagnostics for first-line immune checkpoint inhibitor treatment in non-small cell lung cancer. Transl Lung Cancer Res 2018;7:S95-9.

16. Demaria $\mathrm{S}, \mathrm{Ng}$ B, Devitt $\mathrm{ML}$, et al. Ionizing radiation inhibition of distant untreated tumors (abscopal effect) 
is immune mediated. Int J Radiat Oncol Biol Phys 2004;58:862-70.

17. Chakravarty PK, Alfieri A, Thomas EK, et al. Flt3-ligand administration after radiation therapy prolongs survival in a murine model of metastatic lung cancer. Cancer Res 1999;59:6028-32.

18. Shi W, Siemann DW. Augmented antitumor effects of radiation therapy by 4-1BB antibody (BMS-469492) treatment. Anticancer Res 2006;26:3445-53.

19. Golden EB, Pellicciotta I, Demaria S, et al. The convergence of radiation and immunogenic cell death signaling pathways. Front Oncol 2012;2:88.

20. Ebner DK, Tinganelli W, Helm A, et al. The Immunoregulatory Potential of Particle Radiation in Cancer Therapy. Front Immunol 2017;8:99.

21. Hallahan DE, Virudachalam S, Sherman ML, et al. Tumor necrosis factor gene expression is mediated by protein kinase $\mathrm{C}$ following activation by ionizing radiation. Cancer Res 1991;51:4565-9.

22. Apetoh L, Ghiringhelli F, Tesniere A, et al. Toll-like receptor 4-dependent contribution of the immune system to anticancer chemotherapy and radiotherapy. Nat Med 2007;13:1050-9.

23. Weichselbaum RR, Liang H, Deng L, et al. Radiotherapy and immunotherapy: a beneficial liaison? Nat Rev Clin Oncol 2017;14:365-79.

24. Abuodeh Y, Venkat P, Kim S. Systematic review of case reports on the abscopal effect. Curr Probl Cancer 2016;40:25-37.

25. Wennerberg E, Lhuillier C, Vanpouille-Box C, et al. Barriers to Radiation-Induced In Situ Tumor Vaccination. Front Immunol 2017;8:229.

26. Gong X, Li X, Jiang T, et al. Combined Radiotherapy and Anti-PD-L1 Antibody Synergistically Enhances Antitumor Effect in Non-Small Cell Lung Cancer. J Thorac Oncol 2017;12:1085-97.

27. Kwon ED, Foster BA, Hurwitz AA, et al. Elimination of residual metastatic prostate cancer after surgery and adjunctive cytotoxic $\mathrm{T}$ lymphocyte-associated antigen 4 (CTLA-4) blockade immunotherapy. Proc Natl Acad Sci U S A 1999;96:15074-9.

28. Heigener DF, Reck M. Immune Checkpoint Inhibition in Non-metastatic Non-small Cell Lung Cancer: Chance for Cure? Drugs 2019;79:1937-45.

29. Wirsdörfer F, Jendrossek V. The Role of Lymphocytes in Radiotherapy-Induced Adverse Late Effects in the Lung. Front Immunol 2016;7:591.

30. Piccinini AM, Midwood KS. DAMPening inflammation by modulating TLR signalling. Mediators Inflamm. 2010;2010:672395.

31. Sprung CN, Forrester HB, Siva S, et al. Immunological markers that predict radiation toxicity. Cancer Lett 2015;368:191-7.

32. Du S, Zhou L, Alexander GS, et al. PD-1 Modulates Radiation-Induced Cardiac Toxicity through Cytotoxic T Lymphocytes. J Thorac Oncol 2018;13:510-20.

33. Myers CJ, Lu B. Decreased Survival After Combining Thoracic Irradiation and an Anti-PD-1 Antibody Correlated With Increased T-cell Infiltration Into Cardiac and Lung Tissues. Int J Radiat Oncol Biol Phys 2017;99:1129-36.

34. Lee HJ Jr, Zeng J, Rengan R. Proton beam therapy and immunotherapy: an emerging partnership for immune activation in non-small cell lung cancer. Transl Lung Cancer Res 2018;7:180-8.

35. Darby SC, Cutter DJ, Boerma M, et al. Radiation-related heart disease: current knowledge and future prospects. Int J Radiat Oncol Biol Phys 2010;76:656-65.

36. Gomez DR, Yusuf SW, Munsell MF, et al. Prospective exploratory analysis of cardiac biomarkers and electrocardiogram abnormalities in patients receiving thoracic radiation therapy with high-dose heart exposure. J Thorac Oncol 2014;9:1554-60.

37. Grabie N, Gotsman I, DaCosta R, et al. Endothelial programmed death-1 ligand 1 (PD-L1) regulates CD8+ T-cell mediated injury in the heart. Circulation 2007;116:2062-71.

38. Larkin J, Chiarion-Sileni V, Gonzalez R, et al. Combined Nivolumab and Ipilimumab or Monotherapy in Untreated Melanoma. N Engl J Med 2015;373:23-34.

39. Formenti SC, Demaria S. Combining radiotherapy and cancer immunotherapy: a paradigm shift. J Natl Cancer Inst 2013;105:256-65.

40. Nakamura N, Kusunoki Y, Akiyama M. Radiosensitivity of CD4 or CD8 positive human T-lymphocytes by an in vitro colony formation assay. Radiat Res 1990;123:224-7.

41. Multhoff G, Rödel F, Pockley AG, et al. Frontiers research topic: radiation-induced effects and the immune system. Front Oncol 2013;3:55.

42. Ellsworth SG. Field size effects on the risk and severity of treatment-induced lymphopenia in patients undergoing radiation therapy for solid tumors. Adv Radiat Oncol 2018;3:512-9.

43. Tang C, Liao Z, Gomez D, et al. Lymphopenia association with gross tumor volume and lung V5 and its effects on non-small cell lung cancer patient outcomes. Int J Radiat 
Oncol Biol Phys 2014;89:1084-91.

44. Campian JL, Ye X, Brock M, et al. Treatment-related lymphopenia in patients with stage III non-small-cell lung cancer. Cancer Invest 2013;31:183-8.

45. McCoy MJ, Lake RA, van der Most RG, et al. Postchemotherapy T-cell recovery is a marker of improved survival in patients with advanced thoracic malignancies. Br J Cancer 2012;107:1107-15.

46. Karantanos T, Karanika S, Seth B, et al. The absolute lymphocyte count can predict the overall survival of patients with non-small cell lung cancer on nivolumab: a clinical study. Clin Transl Oncol 2019;21:206-12.

47. Grills IS, Yan D, Martinez AA, et al. Potential for reduced toxicity and dose escalation in the treatment of inoperable non-small-cell lung cancer: a comparison of intensitymodulated radiation therapy (IMRT), 3D conformal radiation, and elective nodal irradiation. Int J Radiat Oncol Biol Phys 2003;57:875-90.

48. Christian JA, Bedford JL, Webb S, et al. Comparison of inverse-planned three-dimensional conformal radiotherapy and intensity-modulated radiotherapy for non-small-cell lung cancer. Int J Radiat Oncol Biol Phys 2007;67:735-41.

49. Murshed H, Liu HH, Liao Z, et al. Dose and volume reduction for normal lung using intensity-modulated radiotherapy for advanced-stage non-small-cell lung cancer. Int J Radiat Oncol Biol Phys 2004;58:1258-67.

50. Sher DJ, Koshy M, Liptay MJ, et al. Influence of conformal radiotherapy technique on survival after chemoradiotherapy for patients with stage III non-small cell lung cancer in the National Cancer Data Base. Cancer 2014;120:2060-8.

51. Yom SS, Liao Z, Liu HH, et al. Initial evaluation of treatment-related pneumonitis in advanced-stage nonsmall-cell lung cancer patients treated with concurrent chemotherapy and intensity-modulated radiotherapy. Int J Radiat Oncol Biol Phys 2007;68:94-102.

52. Liao ZX, Komaki RR, Thames HD Jr, et al. Influence of technologic advances on outcomes in patients with unresectable, locally advanced non-small-cell lung cancer receiving concomitant chemoradiotherapy. Int J Radiat Oncol Biol Phys 2010;76:775-81.

53. Liu HH, Wang X, Dong L, et al. Feasibility of sparing lung and other thoracic structures with intensitymodulated radiotherapy for non-small-cell lung cancer. Int J Radiat Oncol Biol Phys 2004;58:1268-79.

54. Meng LL, Feng LC, Wang YL, et al. Dosimetric comparison between helical tomotherapy and intensitymodulated radiation therapy plans for non-small cell lung cancer. Chin Med J (Engl) 2011;124:1667-71.

55. Zhu Z, Fu X. The radiation techniques of tomotherapy $\&$ intensity-modulated radiation therapy applied to lung cancer. Transl Lung Cancer Res 2015;4:265-74.

56. Wang SL, Liao Z, Vaporciyan AA, et al. Investigation of clinical and dosimetric factors associated with postoperative pulmonary complications in esophageal cancer patients treated with concurrent chemoradiotherapy followed by surgery. Int J Radiat Oncol Biol Phys 2006;64:692-9.

57. Wang S, Liao Z, Wei X, et al. Analysis of clinical and dosimetric factors associated with treatment-related pneumonitis (TRP) in patients with non-small-cell lung cancer (NSCLC) treated with concurrent chemotherapy and three-dimensional conformal radiotherapy (3D-CRT). Int J Radiat Oncol Biol Phys 2006;66:1399-407.

58. Cho O, Oh YT, Chun M, et al. Radiation-related lymphopenia as a new prognostic factor in limited-stage small cell lung cancer. Tumour Biol 2016;37:971-8.

59. Zhang X, Li Y, Pan X, et al. Intensity-modulated proton therapy reduces the dose to normal tissue compared with intensity-modulated radiation therapy or passive scattering proton therapy and enables individualized radical radiotherapy for extensive stage IIIB non-small-cell lung cancer: a virtual clinical study. Int J Radiat Oncol Biol Phys 2010;77:357-66.

60. Nichols RC, Huh SN, Henderson RH, et al. Proton radiation therapy offers reduced normal lung and bone marrow exposure for patients receiving dose-escalated radiation therapy for unresectable stage iii non-smallcell lung cancer: a dosimetric study. Clin Lung Cancer 2011;12:252-7.

61. Higgins KA, O'Connell K, Liu Y, et al. National Cancer Database Analysis of Proton Versus Photon Radiation Therapy in Non-Small Cell Lung Cancer. Int J Radiat Oncol Biol Phys 2017;97:128-37.

62. Liao Z, Lee JJ, Komaki R, et al. Bayesian Adaptive Randomization Trial of Passive Scattering Proton Therapy and Intensity-Modulated Photon Radiotherapy for Locally Advanced Non-Small-Cell Lung Cancer. J Clin Oncol 2018;36:1813-22.

63. Chang JY, Zhang X, Knopf A, et al. Consensus Guidelines for Implementing Pencil-Beam Scanning Proton Therapy for Thoracic Malignancies on Behalf of the PTCOG Thoracic and Lymphoma Subcommittee. Int J Radiat Oncol Biol Phys 2017;99:41-50.

64. Fang P, Shiraishi Y, Verma V, et al. Lymphocyte-Sparing Effect of Proton Therapy in Patients with Esophageal Cancer Treated with Definitive Chemoradiation. Int J Part 
Ther 2018;4:23-32.

65. Shiraishi Y, Fang P, Xu C, et al. Severe lymphopenia during neoadjuvant chemoradiation for esophageal cancer: A propensity matched analysis of the relative risk of proton versus photon-based radiation therapy. Radiother Oncol 2018;128:154-60.

66. Gameiro SR, Malamas AS, Bernstein MB, et al. Tumor Cells Surviving Exposure to Proton or Photon Radiation Share a Common Immunogenic Modulation Signature, Rendering Them More Sensitive to T Cell-Mediated Killing. Int J Radiat Oncol Biol Phys 2016;95:120-30.

67. Palucka K, Banchereau J. Cancer immunotherapy via dendritic cells. Nat Rev Cancer 2012;12:265-77.

68. Ohkubo Y, Iwakawa M, Seino K, et al. Combining carbon ion radiotherapy and local injection of alphagalactosylceramide-pulsed dendritic cells inhibits lung metastases in an in vivo murine model. Int J Radiat Oncol Biol Phys 2010;78:1524-31.

69. Ando K, Fujita H, Hosoi A, et al. Intravenous dendritic cell administration enhances suppression of lung metastasis induced by carbon-ion irradiation. J Radiat Res 2017;58:446-55.

70. Shimokawa T, Ma L, Ando K, et al. The Future of Combining Carbon-Ion Radiotherapy with Immunotherapy: Evidence and Progress in Mouse Models. International Journal of Particle Therapy 2016;3:61-70.

71. Yuan S, Sun X, Li M, et al. A randomized study of involved-field irradiation versus elective nodal irradiation in combination with concurrent chemotherapy for inoperable stage III nonsmall cell lung cancer. Am J Clin Oncol 2007;30:239-44.

72. Chen M, Bao Y, Ma HL, et al. Involved-field radiotherapy versus elective nodal irradiation in combination with concurrent chemotherapy for locally advanced non-small cell lung cancer: a prospective randomized study. Biomed Res Int 2013;2013:371819.

73. Li R, Yu L, Lin S, et al. Involved field radiotherapy (IFRT) versus elective nodal irradiation (ENI) for locally advanced non-small cell lung cancer: a meta-analysis of incidence of elective nodal failure (ENF). Radiat Oncol 2016;11:124.

74. Fernandes AT, Shen J, Finlay J, et al. Elective nodal irradiation (ENI) vs. involved field radiotherapy (IFRT) for locally advanced non-small cell lung cancer (NSCLC): A comparative analysis of toxicities and clinical outcomes. Radiother Oncol 2010;95:178-84.

75. Rosenzweig KE, Sura S, Jackson A, et al. Involved-field radiation therapy for inoperable non small-cell lung cancer. J Clin Oncol 2007;25:5557-61.
76. Senan S, Burgers S, Samson MJ, et al. Can elective nodal irradiation be omitted in stage III non-small-cell lung cancer? Analysis of recurrences in a phase II study of induction chemotherapy and involved-field radiotherapy. Int J Radiat Oncol Biol Phys 2002;54:999-1006.

77. O'Donnell JS, Teng MWL, Smyth MJ. Cancer immunoediting and resistance to $\mathrm{T}$ cell-based immunotherapy. Nat Rev Clin Oncol 2019;16:151-67.

78. Venkatesulu BP, Mallick S, Lin SH, et al. A systematic review of the influence of radiation-induced lymphopenia on survival outcomes in solid tumors. Crit Rev Oncol Hematol 2018;123:42-51.

79. Marciscano AE, Ghasemzadeh A, Nirschl TR, et al. Elective Nodal Irradiation Attenuates the Combinatorial Efficacy of Stereotactic Radiation Therapy and Immunotherapy. Clin Cancer Res 2018;24:5058-71.

80. Withers HR, Suwinski R. Radiation dose response for subclinical metastases. Semin Radiat Oncol 1998;8:224-8.

81. Withers HR, Peters LJ, Taylor JM. Dose-response relationship for radiation therapy of subclinical disease. Int J Radiat Oncol Biol Phys 1995;31:353-9.

82. Zhao L, Chen M, Ten Haken R, et al. Three-dimensional conformal radiation may deliver considerable dose of incidental nodal irradiation in patients with early stage node-negative non-small cell lung cancer when the tumor is large and centrally located. Radiother Oncol 2007;82:153-9.

83. Xia F, Zhou L, Yang X, et al. Is a clinical target volume (CTV) necessary for locally advanced non-small cell lung cancer treated with intensity-modulated radiotherapy? -a dosimetric evaluation of three different treatment plans. J Thorac Dis 2017;9:5194-202.

84. Liang X, Yu H, Yu R, et al. Efficacy of the smaller target volume for stage III non-small cell lung cancer treated with intensity-modulated radiotherapy. Mol Clin Oncol 2015;3:1172-6.

85. Kilburn JM, Lucas JT, Soike MH, et al. Is a Clinical Target Volume (CTV) Necessary in the Treatment of Lung Cancer in the Modern Era Combining 4-D Imaging and Image-guided Radiotherapy (IGRT)? Cureus 2016;8:e466.

86. Perez CA, Stanley K, Rubin P, et al. A prospective randomized study of various irradiation doses and fractionation schedules in the treatment of inoperable non-oat-cell carcinoma of the lung. Preliminary report by the Radiation Therapy Oncology Group. Cancer 1980;45:2744-53.

87. Bradley JD, Paulus R, Komaki R, et al. Standard-dose versus high-dose conformal radiotherapy with concurrent 
and consolidation carboplatin plus paclitaxel with or without cetuximab for patients with stage IIIA or IIIB non-small-cell lung cancer (RTOG 0617): a randomised, two-by-two factorial phase 3 study. Lancet Oncol 2015;16:187-99.

88. Mauguen A, Le Pechoux C, Saunders MI, et al. Hyperfractionated or accelerated radiotherapy in lung cancer: an individual patient data meta-analysis. J Clin Oncol 2012;30:2788-97.

89. Saunders M, Dische S, Barrett A, et al. Continuous hyperfractionated accelerated radiotherapy (CHART) versus conventional radiotherapy in non-small-cell lung cancer: a randomised multicentre trial. CHART Steering Committee. Lancet 1997;350:161-5.

90. Ball D, Bishop J, Smith J, et al. A randomised phase III study of accelerated or standard fraction radiotherapy with or without concurrent carboplatin in inoperable non-small cell lung cancer: final report of an Australian multi-centre trial. Radiother Oncol 1999;52:129-36.

91. Schild SE, Stella PJ, Geyer SM, et al. Phase III trial comparing chemotherapy plus once-daily or twice-daily radiotherapy in Stage III non-small-cell lung cancer. Int J Radiat Oncol Biol Phys 2002;54:370-8.

92. Belani CP, Wang W, Johnson DH, et al. Phase III study of the Eastern Cooperative Oncology Group (ECOG 2597): induction chemotherapy followed by either standard thoracic radiotherapy or hyperfractionated accelerated radiotherapy for patients with unresectable stage IIIA and B non-small-cell lung cancer. J Clin Oncol 2005;23:3760-7.

93. Baumann M, Herrmann T, Koch R, et al. Final results of the randomized phase III CHARTWEL-trial (ARO 97-1) comparing hyperfractionated-accelerated versus conventionally fractionated radiotherapy in non-small cell lung cancer (NSCLC). Radiother Oncol 2011;100:76-85.

94. Stratton JA, Byfield PE, Byfield JE, et al. A comparison of the acute effects of radiation therapy, including or excluding the thymus, on the lymphocyte subpopulations of cancer patients. J Clin Invest 1975;56:88-97.

95. Sellins KS, Cohen JJ. Gene induction by gammairradiation leads to DNA fragmentation in lymphocytes. J Immunol 1987;139:3199-206.

96. Galluzzi L, Buque A, Kepp O, et al. Immunogenic cell death in cancer and infectious disease. Nat Rev Immunol 2017;17:97-111.

97. Wu Q, Allouch A, Martins I, et al. Modulating Both Tumor Cell Death and Innate Immunity Is Essential for Improving Radiation Therapy Effectiveness. Front
Immunol 2017;8:613.

98. Eckert F, Zwirner K, Boeke S, et al. Rationale for Combining Radiotherapy and Immune Checkpoint Inhibition for Patients With Hypoxic Tumors. Front Immunol 2019;10:407.

99. Ostrand-Rosenberg S, Horn LA, Ciavattone NG. Radiotherapy Both Promotes and Inhibits MyeloidDerived Suppressor Cell Function: Novel Strategies for Preventing the Tumor-Protective Effects of Radiotherapy. Front Oncol 2019;9:215.

100. Morisada M, Clavijo PE, Moore E, et al. PD-1 blockade reverses adaptive immune resistance induced by highdose hypofractionated but not low-dose daily fractionated radiation. Oncoimmunology 2018;7:e1395996.

101. Vanpouille-Box C, Alard A, Aryankalayil MJ, et al. DNA exonuclease Trex1 regulates radiotherapy-induced tumour immunogenicity. Nat Commun 2017;8:15618.

102.Lo SS, Fakiris AJ, Chang EL, et al. Stereotactic body radiation therapy: a novel treatment modality. Nat Rev Clin Oncol 2010;7:44-54.

103. Gough MJ, Young K, Crittenden M. The impact of the myeloid response to radiation therapy. Clin Dev Immunol 2013;2013:281958.

104. Russell JS, Brown JM. The irradiated tumor microenvironment: role of tumor-associated macrophages in vascular recovery. Front Physiol 2013;4:157.

105.Wu Q, Allouch A, Martins I, et al. Macrophage biology plays a central role during ionizing radiation-elicited tumor response. Biomed J 2017;40:200-11.

106. Klug F, Prakash H, Huber PE, et al. Low-dose irradiation programs macrophage differentiation to an iNOS(+)/ M1 phenotype that orchestrates effective T cell immunotherapy. Cancer Cell 2013;24:589-602.

107.Prakash H, Klug F, Nadella V, et al. Low doses of gamma irradiation potentially modifies immunosuppressive tumor microenvironment by retuning tumor-associated macrophages: lesson from insulinoma. Carcinogenesis 2016;37:301-13.

108. Filatenkov A, Baker J, Mueller AM, et al. Ablative Tumor Radiation Can Change the Tumor Immune Cell Microenvironment to Induce Durable Complete Remissions. Clin Cancer Res 2015;21:3727-39.

109. Lan J, Li R, Yin LM, et al. Targeting Myeloidderived Suppressor Cells and Programmed Death Ligand 1 Confers Therapeutic Advantage of Ablative Hypofractionated Radiation Therapy Compared With Conventional Fractionated Radiation Therapy. Int J Radiat Oncol Biol Phys 2018;101:74-87. 
110.Bernichon E, Vallard A, Wang Q, et al. Genomic alterations and radioresistance in breast cancer: an analysis of the ProfiLER protocol. Ann Oncol 2017;28:2773-9.

111. Ahmed KA, Berglund AE, Welsh EA, et al. The radiosensitivity of brain metastases based upon primary histology utilizing a multigene index of tumor radiosensitivity. Neuro Oncol 2017;19:1145-6.

112. Kerns SL, Fachal L, Dorling L, et al. Radiogenomics Consortium Genome-Wide Association Study Metaanalysis of Late Toxicity after Prostate Cancer Radiotherapy. J Natl Cancer Inst 2020;112:179-90.

113. Pavlopoulou A, Bagos PG, Koutsandrea V, et al. Molecular determinants of radiosensitivity in normal and tumor tissue: A bioinformatic approach. Cancer Lett 2017;403:37-47.

114. King TC, Estalilla OC, Safran H. Role of p53 and p16 gene alterations in determining response to concurrent paclitaxel and radiation in solid tumor. Semin Radiat Oncol 1999;9:4-11.

115.Sak A, Stueben G, Groneberg M, et al. Targeting of Rad51-dependent homologous recombination: implications for the radiation sensitivity of human lung cancer cell lines. Br J Cancer 2005;92:1089-97.

116. Edvardsen H, Tefre T, Jansen L, et al. Linkage disequilibrium pattern of the ATM gene in breast cancer patients and controls; association of SNPs and haplotypes to radio-sensitivity and post-lumpectomy local recurrence. Radiat Oncol 2007;2:25.

117.Lee YS, Oh JH, Yoon S, et al. Differential gene expression profiles of radioresistant non-small-cell lung cancer cell lines established by fractionated irradiation: tumor protein p53-inducible protein 3 confers sensitivity to ionizing radiation. Int J Radiat Oncol Biol Phys 2010;77:858-66.

118.Jin JY, Wang W, Ten Haken RK, et al. Use a survival model to correlate single-nucleotide polymorphisms of DNA repair genes with radiation dose-response in patients with non-small cell lung cancer. Radiother Oncol 2015;117:77-82.

119.Lim YJ, Chang JH, Kim HJ, et al. Superior Treatment Response and In-field Tumor Control in Epidermal Growth Factor Receptor-mutant Genotype of Stage III Nonsquamous Non-Small cell Lung Cancer Undergoing Definitive Concurrent Chemoradiotherapy. Clin Lung Cancer 2017;18:e169-78.

120.Das AK, Sato M, Story MD, et al. Non-small-cell lung cancers with kinase domain mutations in the epidermal growth factor receptor are sensitive to ionizing radiation. Cancer Res 2006;66:9601-8.
121. Yagishita S, Horinouchi H, Katsui Taniyama T, et al. Epidermal growth factor receptor mutation is associated with longer local control after definitive chemoradiotherapy in patients with stage III nonsquamous non-small-cell lung cancer. Int J Radiat Oncol Biol Phys 2015;91:140-8.

122.Johung KL, Yao X, Li F, et al. A clinical model for identifying radiosensitive tumor genotypes in non-small cell lung cancer. Clin Cancer Res 2013;19:5523-32.

123. De Ruyck K, Van Eijkeren M, Claes K, et al. TGFbeta1 polymorphisms and late clinical radiosensitivity in patients treated for gynecologic tumors. Int J Radiat Oncol Biol Phys 2006;65:1240-8.

124. Alsbeih G, El-Sebaie M, Al-Harbi N, et al. Radiosensitivity of human fibroblasts is associated with amino acid substitution variants in susceptible genes and correlates with the number of risk alleles. Int J Radiat Oncol Biol Phys 2007;68:229-35.

125. Kelsey CR, Jackson L, Langdon S, et al. A polymorphism within the promoter of the TGFbetal gene is associated with radiation sensitivity using an objective radiologic endpoint. Int J Radiat Oncol Biol Phys 2012;82:e247-55.

126. Kim EH, Park AK, Dong SM, et al. Global analysis of $\mathrm{CpG}$ methylation reveals epigenetic control of the radiosensitivity in lung cancer cell lines. Oncogene 2010;29:4725-31.

127.Pugh TJ, Keyes M, Barclay L, et al. Sequence variant discovery in DNA repair genes from radiosensitive and radiotolerant prostate brachytherapy patients. Clin Cancer Res 2009;15:5008-16.

128. Souchek JJ, Baine MJ, Lin C, et al. Unbiased analysis of pancreatic cancer radiation resistance reveals cholesterol biosynthesis as a novel target for radiosensitisation. Br J Cancer 2014;111:1139-49.

129. Torres-Roca JF, Eschrich S, Zhao H, et al. Prediction of radiation sensitivity using a gene expression classifier. Cancer Res 2005;65:7169-76.

130.Amundson SA, Do KT, Vinikoor LC, et al. Integrating global gene expression and radiation survival parameters across the 60 cell lines of the National Cancer Institute Anticancer Drug Screen. Cancer Res 2008;68:415-24.

131. Eschrich S, Zhang H, Zhao H, et al. Systems biology modeling of the radiation sensitivity network: a biomarker discovery platform. Int J Radiat Oncol Biol Phys 2009;75:497-505.

132. Eschrich SA, Pramana J, Zhang H, et al. A gene expression model of intrinsic tumor radiosensitivity: prediction of response and prognosis after chemoradiation. Int J Radiat 
Oncol Biol Phys 2009;75:489-96.

133. Scott JG, Berglund A, Schell MJ, et al. A genomebased model for adjusting radiotherapy dose (GARD): a retrospective, cohort-based study. Lancet Oncol 2017;18:202-11.

134. Ahmed KA, Liveringhouse CL, Mills MN, et al. Utilizing the genomically adjusted radiation dose (GARD) to personalize adjuvant radiotherapy in triple negative breast cancer management. EBioMedicine 2019;47:163-9.

135. Oliver DE, Mohammadi H, Figura N, et al. Novel Genomic-Based Strategies to Personalize Lymph Node Radiation Therapy. Semin Radiat Oncol 2019;29:111-25.

136.Zhang H, Wang M, Shi T, et al. Genetic polymorphisms of PAI- 1 and PAR-1 are associated with acute normal tissue toxicity in Chinese rectal cancer patients treated with pelvic radiotherapy. Onco Targets Ther 2015;8:2291-301.

137. Borchiellini D, Etienne-Grimaldi MC, Bensadoun RJ, et al. Candidate apoptotic and DNA repair gene approach confirms involvement of ERCC1, ERCC5, TP53 and MDM2 in radiation-induced toxicity in head and neck cancer. Oral Oncol 2017;67:70-6.

138. Toffoli G, Cecchin E, Gasparini G, et al. Genotype-driven phase I study of irinotecan administered in combination with fluorouracil/leucovorin in patients with metastatic colorectal cancer. J Clin Oncol 2010;28:866-71.

Cite this article as: Guo T, Zou L, Ni J, Chu X, Zhu Z. Radiotherapy for unresectable locally advanced non-small cell lung cancer: a narrative review of the current landscape and future prospects in the era of immunotherapy. Transl Lung Cancer Res 2020;9(5):2097-2112. doi: 10.21037/tlcr-20-511
139.Zhu J, Li X, Shen Y, et al. Genotype-driven phase I study of weekly irinotecan in combination with capecitabinebased neoadjuvant chemoradiation for locally advanced rectal cancer. Radiother Oncol 2018;129:143-8.

140.Innocenti F, Schilsky RL, Ramirez J, et al. Dose-finding and pharmacokinetic study to optimize the dosing of irinotecan according to the UGT1A1 genotype of patients with cancer. J Clin Oncol 2014;32:2328-34.

141. Sharabi AB, Lim M, DeWeese TL, et al. Radiation and checkpoint blockade immunotherapy: radiosensitisation and potential mechanisms of synergy. Lancet Oncol 2015;16:e498-509.

142.Luke JJ, Lemons JM, Karrison TG, et al. Safety and Clinical Activity of Pembrolizumab and Multisite Stereotactic Body Radiotherapy in Patients With Advanced Solid Tumors. J Clin Oncol 2018;36:1611-8.

143. Chicas-Sett R, Morales-Orue I, Castilla-Martinez J, et al. Stereotactic Ablative Radiotherapy Combined with Immune Checkpoint Inhibitors Reboots the Immune Response Assisted by Immunotherapy in Metastatic Lung Cancer: A Systematic Review. Int J Mol Sci 2019;20.

144. Chandra RA, Wilhite TJ, Balboni TA, et al. A systematic evaluation of abscopal responses following radiotherapy in patients with metastatic melanoma treated with ipilimumab. Oncoimmunology 2015;4:e1046028. 